\title{
Interictal brain activity differs in migraine with and without aura: resting state fMRI study
}

Péter Faragó ${ }^{1}$, Bernadett Tuka ${ }^{1,2}$, Eszter Tóth ${ }^{1}$, Nikoletta Szabó ${ }^{1,3}$, András Király ${ }^{1}$, Gergő Csete ${ }^{1}$, Délia Szok ${ }^{1}$, János Tajti ${ }^{1}$, Árpád Párdutz ${ }^{1}$, László Vécsei ${ }^{1,2}$ and Zsigmond Tamás Kincses ${ }^{1,3^{*}}$

\begin{abstract}
Background: Migraine is one of the most severe primary headache disorders. The nature of the headache and the associated symptoms during the attack suggest underlying functional alterations in the brain. In this study, we examined amplitude, the resting state fMRI fluctuation in migraineurs with and without aura (MWA, MWoA respectively) and healthy controls.

Methods: Resting state functional MRI images and T1 high-resolution images were acquired from all participants. For data analysis we compared the groups (MWA-Control, MWA-MWoA, MWoA-Control). The resting state networks were identified by MELODIC. The mean time courses of the networks were identified for each participant for all networks. The time-courses were decomposed into five frequency bands by discrete wavelet decomposition. The amplitude of the frequency-specific activity was compared between groups. Furthermore, the preprocessed resting state images were decomposed by wavelet analysis into five specific frequency bands voxel-wise. The voxel-wise amplitudes were compared between groups by non-parametric permutation test.

Results: In the MWA-Control comparison the discrete wavelet decomposition found alterations in the lateral visual network. Higher activity was measured in the MWA group in the highest frequency band $(0.16-0.08 \mathrm{~Hz})$.

In case of the MWA-MWoA comparison all networks showed higher activity in the $0.08-0.04 \mathrm{~Hz}$ frequency range in MWA, and the lateral visual network in in higher frequencies.

In MWoA-Control comparison only the default mode network revealed decreased activity in MWoA group in the 0. $08-0.04 \mathrm{~Hz}$ band.

The voxel-wise frequency specific analysis of the amplitudes found higher amplitudes in MWA as compared to MWoA in the in fronto-parietal regions, anterior cingulate cortex and cerebellum.

Discussion: The amplitude of the resting state fMRI activity fluctuation is higher in MWA than in MWoA. These results are in concordance with former studies, which found cortical hyperexcitability in MWA.
\end{abstract}

Keywords: ALFF, Frequency analysis, Migraine, Migraine with aura, MRI

\footnotetext{
*Correspondence: kincses.zsigmond.tamas@med.u-szeged.hu; http://www. nepsy.szote.u-szeged.hu/ kincsesz

${ }^{1}$ Department of Neurology, Neuroimaging Research Group, Albert

Szent-Györgyi, Clinical Center, University of Szeged, Semmelweis u. 6, H-6725

Szeged, Hungary

${ }^{3}$ International Clinical Research Center, St. Anne's University Hospital Brno,

Brno, Czech Republic

Full list of author information is available at the end of the article
} 


\section{Background}

Migraine is a common disabling disease, affecting about $10 \%$ of the population [1]. While not life threatening, it has a significant effect on the quality of life. Two major forms of the disease present with or without transient, focal neurological symptoms, called aura [2]. While the pathomechanism of migraine is not entirely understood, the two subgroups of the disease thought to have different background [3, 4]. It was suggested that cortical spreading depression, a slow depolarization wave traveling anteriorly in the brain is responsible for the aura symptoms and occur exclusively in migraine with aura (MWA) $[5,6]$. Cortical hyperexcitability was mentioned as the potential trigger of migraine [7-12] and this hyperexcitability is also present in MWA more robustly [13].

Information about the resting brain activity can be noninvasively gathered by BOLD fMRI. Traditionally fMRI studies compare signal in various phases of a task, but acquiring BOLD signal in rest, allows the studying of resting brain activity fluctuations. Interestingly, remote areas show synchronous activity, which renders resting state activity into functional networks $[14,15]$.

Several studies investigated the activity of the resting state functional networks in migraine and found various alterations of networks that are implicated in pain processing [16-19]. Furthermore, a few studies investigated patients with MWA and migraine without aura (MWoA) and reported various aspects of altered connectivity in the subgroups of the disease [20-25]. Remarkably some of the studies found increased functional connectivity, in comparison to decreased functional connectivity in migraine.

Most of the resting state fMRI studies investigated the connectivity between various regions and therefore build on the coherent activity in spatially distributed networks. The variation in the frequency and the amplitude of the resting state BOLD signal is usually neglected. The BOLD resting state fluctuation is a low frequency fluctuation $[14,15]$. To filter out the non-neural noise from the raw BOLD time courses most of the studies apply filters [16, 26, 27]. However, neural signal could be detected also in the higher frequencies [28]. Furthermore, a few recent studies started to analyse the amplitude of the low frequency fluctuation of the resting state signals $[29,30]$ offering a unique insight into the resting brain activity in various diseases but not in migraine.

Based on the above described premises, the increased cortical excitability in migraine might be related to the frequency specific alteration of amplitude of the resting BOLD activity. MWA and MWoA being different in respect to cortical excitability might also appear in the differential frequency spectrum of the resting brain activity in the two subgroups of the disease.
In the current study we investigated the resting state BOLD fluctuations in migraine with special focus on the amplitude and the frequency of the activity.

\section{Methods \\ Participants}

Fifty-three patients with migraine were recruited into this study from the Headache Outpatients Clinic, at the Department of Neurology, University of Szeged. All of the patients were diagnosed with episodic migraine and were scanned during the interictal phase, having had the the scanning at least one week to the last attack. The diagnosis set up by the International Classification of Headache Disorders [2]. Eighteen patients suffered from MWA (17 visual aura, 1 sensory aura), the other 35 patients never experienced aura. Patients had no other neurological or psychiatric disorders.

Thirty-two healthy volunteers were recruited. None of the controls had any records of any neurological or psychiatric disorders. For demographic data of patients and controls, see Table 1.

The study was approved by the ethics committee of the University of Szeged and all study participants gave written informed consent in accordance with the Declaration of Helsinki (authority number: 56/2011).

\section{Image acquisition}

The MR imaging was performed on a $1.5 \mathrm{~T}$ GE Signa Excite HDxt MRI Scanner (Milwaukee, WI, USA). The head motion was restricted with foam padding around the head and the noise of the scanner was attenuated with earplugs. For every participants high-resolution T1 weighted images (3D IR-FSPGR: TR/TE/TI: 10.3/4.2/ $450 \mathrm{~ms}$, flip angle: $15^{\circ}$, ASSET: 2, FOV: $25^{*} 25 \mathrm{~cm}$, matrix: 256*256, slice thickness: $1 \mathrm{~mm}$,) and a resting state fMRI protocol with echo-planar imaging technique (TE: $40 \mathrm{~ms}$, TR: $3000 \mathrm{~ms}$, matrix: 64*64 cm, FOV: $30 * 30 \mathrm{~cm}$, slice thickness: $6 \mathrm{~mm}$, flip angle: $90^{\circ}$, NEX: 1 , ASSET: 2,0 Ph, Phases per Loc: 128, volumes: 200) were acquired. Subjects were asked to be awake during the acquisition eyes closed.

\section{Data processing}

All image processing were performed by FMRIB's Software Library (http://www.fmrib.ox.uk/fsl, Oxford, UK) toolkits.

\section{Preprocessing}

The pre-processing was carried out with FEAT (FMRI Expert Analysis Tool). The first two images were removed from all resting state datasets. The non-brain parts were removed using Brain Extraction (BET) [31]. Motion correction (MCFLIRT) [32] were applied in all images and spatially smoothed with Gaussian kernel of 
Table 1 Demographic data ATK/life: estimated headache attacks over lifetime. ATK/years: annual headache attack frequency. There were no significant difference between the groups in terms of age $(p<0.82)$ or gender $(p<0.8)$. There were no significant difference in the disease duration $(p<0.65)$ or allodynia score $(p<0.2)$ between migraine groups. There were significant differences between the two patient group in VAS $(p<0.0 .3)$ and yearly attacks $(p<0.03)$

\begin{tabular}{llll}
\hline & Migraine with aura & Migraine without aura & Healthy \\
\hline$n$ & 18 & 33 & 32 \\
age (years; mean and SD) & $32.1(8)$ & $35.6(8.9)$ & $35.2(11)$ \\
gender (male) & 3 & 3 & 2 \\
Allodynia & $1.6(1.7)$ & $3.2(3)$ & $\mathbf{N A}$ \\
Disease duration (years; mean and SD) & $14.2(8.6)$ & $13.7(9.1)$ & $\mathbf{N A}$ \\
ATK/life (days; mean) & $461(615)$ & $656(626)$ & $\mathbf{N A}$ \\
VAS & $7.6(1.3)$ & $\mathbf{8 . 7 ( 1 . 2 )}$ & $\mathbf{N A}$ \\
ATK/years (days; mead and SD) & $29(26)$ & $55(45.6)$ & $N A$ \\
\hline
\end{tabular}

6 mm FWHM. The high-pass filter cut-off was applied to all functional images (with sigma $100 \mathrm{~s}$ ).

All pre-processed resting state images were registered to standard space (MNI152 T1 image; 2 mm slice thickness) and to their high-resolution T1 images with linear (FLIRT) and then with non-linear registration (FNIRT) [32]. All images were resampled to $4 \mathrm{~mm}$ isovoxels.

\section{Independent component analysis}

Resting state networks were identified by group independent component analysis as implemented in the MELODIC toolbox, part of the FSL software library (FMRIB Software Library, htttp://www.fmrib.ox.uk/fsl) [15]. The individual preprocessed and standard space registered functional images were concatenated after voxel-wise de-meaning and variance normalization. The $4 \mathrm{D}$ data were decomposed into a set of matrices that characterize the underlying processes in the spatial and temporal domains in such a way that spatial matrices are maximally non-Gaussian. The number of components was estimated by applying the Laplace approximation to the Bayesian evidence of a probabilistic principal component model [33]. Spatial maps were processed by using an alternative hypothesis test based on the fitting a Gaussian/ Gamma mixture model to the distribution of voxel intensities within spatial maps and a posterior probability threshold of $p>0.5$. The resulting subject-wise time courses are to be understood as the temporal characteristics of the network activity across the entire spatial map for each individual subject.

\section{Amplitude of resting state activity}

The frequency specific modulation of the amplitude of resting activity was analysed by discrete wavelet decomposition (Wavelet Toolbox of the Matlab software package; MathWorks Inc). The networks' time courses were divided into five consecutive frequency bands (band1: 0.16-0.08 Hz, band2: $0.08-0.04 \mathrm{~Hz}$, band3: $0.04-$
$0.02 \mathrm{~Hz}$; band4: $0.02-0.01 \mathrm{~Hz}$; band5: $0.01-0 \mathrm{~Hz}$ ) using discrete wavelet decomposition. Discrete wavelet decomposition is an implementation of the wavelet transform using a set of predefined wavelet scales and translations and decomposes the signal into mutually orthogonal set of wavelets. Wavelets are brief waves, they are finitely extended and their oscillations decay to zero rapidly, satisfying the admissibility condition:

$\int \Psi(t) d t=0$.

By dilating and translating a "mother" wavelet $(\Psi)$ and a "father" wavelet $(\Phi)\left(\int \Phi(t) d t=1\right)$ a wavelet family can be obtained:

$$
\begin{aligned}
& \Psi_{j k}(t)=\frac{1}{\sqrt{2^{j}}} \Psi\left(\frac{t-2^{j} k}{2^{j}}\right) ; \\
& \Phi_{j k}(t)=\frac{1}{\sqrt{2^{j}}} \Phi\left(\frac{t-2^{j} k}{2^{j}}\right) ;
\end{aligned}
$$

where $\mathrm{j}$ is index of the scale $S_{j}=2^{j}$ and $\mathrm{k}$ indexes the $K=n / 2^{j}$ location in time. The Daubechies wavelet was used as mother wavelet. The analysis by using a halfband filtering, decomposes the data over a hierarchy of scales $\left(S_{j}\right)$. At each scale the data is split into two orthogonal components: details $\left(d_{j k}\right)$ containing the high frequency information and approximations $\left(a_{j k}\right)$ containing the low frequency information [34]. By five levels of decomposition the following frequency bands were derived $0-0.16 \mathrm{~Hz}: 0.16-0.08 \mathrm{~Hz}, 0.08-0.04 \mathrm{~Hz}$, $0.04-0.02 \mathrm{~Hz}, 0.02-0.01 \mathrm{~Hz}$ and $0.01-0 \mathrm{~Hz}$.

To measure the amplitude of the resting activity in the various frequency bands an envelope was fitted to the absolute values of each frequency bands: The minimums of the following function was identified:

$$
f(t)=\frac{d\left(\operatorname{sgn}\left(\frac{d y}{d t}\right)\right)}{d t}
$$

and a linear interpolation of these points were used to 
create an envelope. The envelopes were averaged over time to describe the mean activity in the given frequency band.

The analysis outlined above was applied to (i) the mean activity of the ICA identified resting state networks and (ii) to the voxel-wise preprocessed fMRI data.

i. To compare the amplitude of the mean network timecourses across groups in the individual frequency bands General Linear Model (GLM) based test was used. Gender and age were included in our analysis as covariate.

ii. For voxel-wise comparison of the amplitude of the resting activity across groups a nonparametric permutation test was performed (5000 permutations) for each frequency bands. The design encoded for group membership, age and gender were used as nuisance variables. For statistical analysis threshold-free cluster enhancement were used (TFCE) and corrected for multiple comparisons (across space) within the permutation framework. Age and gender also included in this analysis as nuisance variable.

Additionally, we performed the voxel wise comparison of the resting state networks' for the non-filtered data also.

\section{Results}

\section{Demographical data}

There were no significant difference between the groups' age or gender distribution. There were no significant differences between the two migraineus group in disease duration.

\section{Amplitude of the activity of the resting state functional networks}

A MELODIC analysis found 33 components in the healthy controls. The artefact components were removed from our analysis based on previous studies $[14,15]$ and five networks were included for analysis: default mode network, right attention network, left attention network, medial visual network, lateral visual network.

\section{MWA vs. healthy controls}

There were no significant differences between the amplitude of the resting activity in MWA vs. healthy controls. While not significant, in the highest frequencies $(0.16-$ $0.08 \mathrm{~Hz}$ ) the amplitude of the resting activity was slightly higher in the left attention network $(p=0.07)$ and in the right attention network $(p=0.059)$ in MWA as compared to healthy controls.
MWA vs. MWoA

The amplitude of the activity was higher $(p<0.05)$ in all examined networks in the $0.08-0.04 \mathrm{~Hz}$ frequency range and in the lateral visual network also in the 0.16$0.08 \mathrm{~Hz}$ frequency band (Fig. 1) in the MWA group. There were no other significant results in any other frequency bands or in case of the non-filtered data.

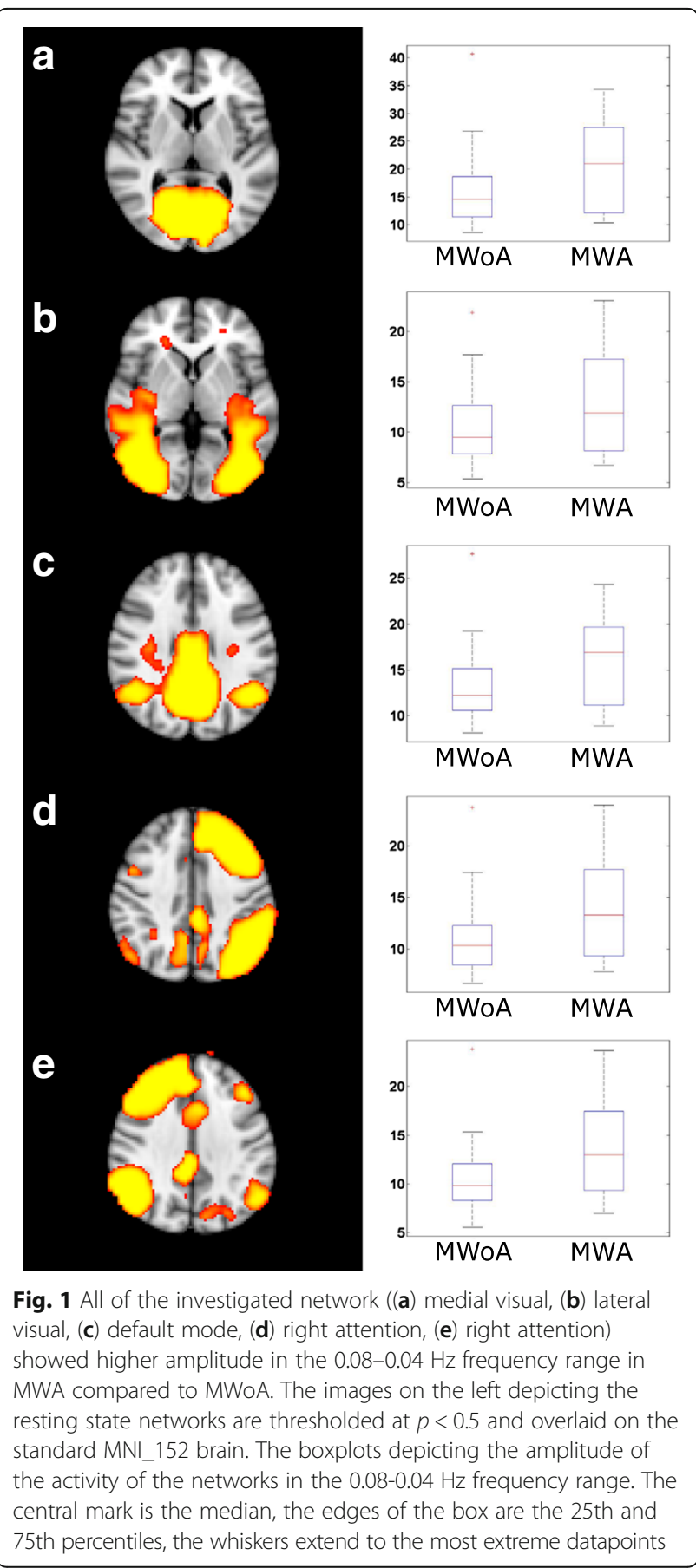




\section{MWoA vs. Healthy controls}

The amplitude of resting activity in the $0.08-0.04 \mathrm{~Hz}$ range was lower in patients without aura in the default mode network $(p<0.05)$ (Fig. 2). There were no other significant results in any other frequency bands or in case of the non-filtered data.

\section{Voxel-wise comparison of the amplitude of resting activity}

The voxel-wise comparison of the amplitude of resting activity showed higher amplitudes $(p<0.05$, corrected for multiple comparisons) in the left parietal lobe in all frequency ranges in MWA compared to MWoA. In addition, in the $0.08-0.04 \mathrm{~Hz}$ frequency band the amplitudes were higher in the bilateral cerebellum, in the left occipital pole and occipito-temporal junction, and a smaller cluster was found in the right inferior parietal lobule. In the 0.04-0.02 Hz frequency band the amplitudes were higher in MWA compared to MWoA in the left inferior parietal lobule, bilateral cerebellum and in the anterior cingulate gyrus. In the $0.02-0.01 \mathrm{~Hz}$ range next to the inferior parietal lobule, occipital pole and cingulate gyral differences amplitudes were found higher in the bilateral frontal lobe around the superior frontal sulcus and precentral gyrus (Table 2 and Fig. 3).

There were no differences between the MWoA and healthy group or between the MWA and control group.

\section{Discussion}

This study identifies novel frequency specific alterations of resting fluctuation of fMRI measured brain activity. Our analysis showed that amplitude of resting state BOLD fluctuation is higher in MWA in the cingulate cortex, superior parietal lobule, cerebellum and bilateral frontal regions compared to MWoA. Furthermore, amplitude of the activity of the resting state networks in the 0.08-0.04 $\mathrm{Hz}$ frequency range was higher in MWA than in MWoA in all examined resting state networks. In MWoA the amplitude of the activity
Table 2 Increased amplitude of resting state activity

fluctuations in MWA as compared to MWoA

\begin{tabular}{lllllll}
\hline & Region & side & $\mathrm{x}$ & $\mathrm{y}$ & $\mathrm{z}$ & $\mathrm{p}<$ \\
\hline 0-0.16 Hz & Inferior parietal lobule & $\mathrm{L}$ & -38 & -82 & 40 & 0.03 \\
0.16-0.08 Hz & Inferior parietal lobule & $\mathrm{L}$ & -34 & -84 & 42 & 0.02 \\
& Cerebellum & $\mathrm{L}$ & -6 & -84 & -40 & 0.04 \\
& Occipito-temporal junction & $\mathrm{L}$ & -58 & -70 & 0 & 0.03 \\
& Occipital pole & $\mathrm{L}$ & -16 & -96 & 14 & 0.05 \\
& Inferior parietal lobule & $\mathrm{R}$ & 14 & -84 & 34 & 0.05 \\
& Cerebellum & $\mathrm{R}$ & 36 & -34 & -52 & 0.05 \\
0.08-0.04 Hzz & Cingulate gyrus & $\mathrm{L}$ & -6 & 14 & -18 & 0.04 \\
& Inferior parietal lobule & $\mathrm{L}$ & -34 & -84 & 42 & 0.03 \\
& Cerebellum & $\mathrm{R}$ & 24 & -84 & -40 & 0.04 \\
& Cerebellum & $\mathrm{L}$ & -14 & -80 & -46 & 0.05 \\
& Inferior parietal lobule & $\mathrm{L}$ & -34 & -84 & 42 & 0.02 \\
& Superior frontal sulcus & $\mathrm{R}$ & 26 & 34 & 44 & 0.04 \\
& Precentral gyrus & $\mathrm{L}$ & -44 & -12 & 62 & 0.04 \\
& Frontal pole & $\mathrm{L}$ & -34 & 52 & -16 & 0.03 \\
& Cingulate gyrus & $\mathrm{L}$ & 0 & -2 & 42 & 0.05 \\
& Occipital lobe & $\mathrm{L}$ & -22 & -92 & 12 & 0.04 \\
$0.04-0.01 \mathrm{~Hz}$ & Inferior parietal lobule & $\mathrm{L}$ & -44 & -60 & 58 & 0.03 \\
& & & & & &
\end{tabular}

fluctuation of the default mode network was lower than in healthy controls.

Several lines of evidence supports the hyperexcitability of the cortex in migraine [7-12]. The amplitude of visual evoked potentials (VEP) were showed higher in migraineurs [11, 34-36]. However, recent reports showed that the VEP measured hyperexcitability predominantly true for migraine with aura [37, 38].

The threshold of transcranial magnetic stimulation evoked phosphenes are also lower in migraineurs and the prevalence of phosphenes are higher [9]. Interestingly, recent metaanalysis pointed out that, similar to the VEP results, this kind of TMS measured
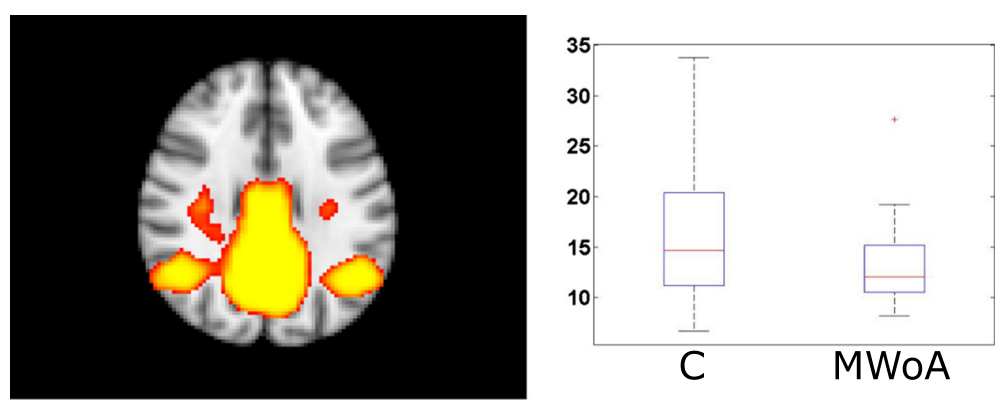

Fig. 2 The default mode network showed higher amplitude in the $0.04-0.02 \mathrm{~Hz}$ in healthy group compared to MWoA. The images on the left depicting the resting state networks are thresholded at $p<0.5$ and overlaid on the standard MNI_152 brain. The boxplots depicting the amplitude of the activity of the networks. The central mark is the median, the edges of the box are the 25th and 75th percentiles, the whiskers extend to the most extreme datapoints 


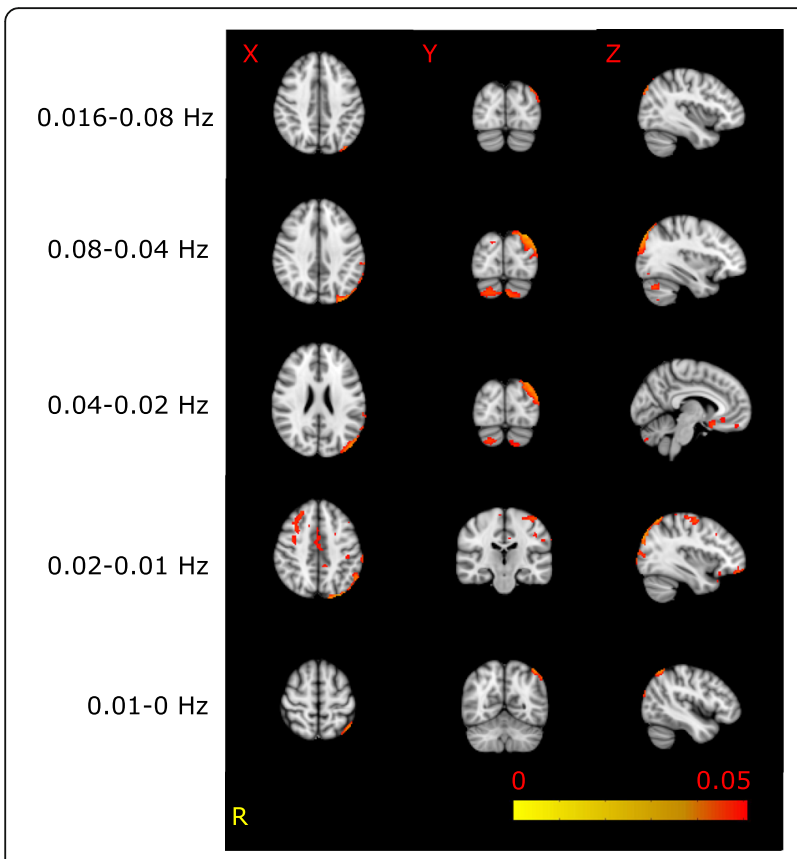

Fig. 3 The frequency specific voxel wise comparison of the amplitude of the resting activity showed higher amplitudes in MWA as compared to MWoA in all freqeuncy ranges. The voxel wise changes in each frequency range. The images are thresholded at $p<0.05$ corrected for multiple comparisons and overlaid on the standard MNI_152 brain. We marked the sidedness with ' $R$ ' as the right side. The $\bar{X}-Y$ - $Z$ letters indicates the axes of the pictures above them

hyperexcitability is not evident for MWoA and only true for patients experiencing aura [39].

Similarly, neuroimaging PET and fMRI studies found higher activation for visual stimuli in migraineurs [40-42], but recent reports showed that patients with aura show higher BOLD response to photic stimuli in contrasts to those migraineurs not experiencing aura symptoms [43, 44].

A possible explanation of such interictal hyperexcitability in migraine might be the higher interictal glutamate/glutamine ratio [45] or the lower gamma-amino butyric acid level [46] in the occipital cortex as shown by MR spectroscopy studies. A recent investigation also showed larger reduction of $\mathrm{N}$-acetyl-aspartate levels in a functional MR spectroscopy study in MWA but not in MWoA [47] indicating a less effective mitochondrial functioning in MWA.

These above mentioned studies, indicating cortical hyperexcitability in MWA are in line with our current results. The amplitude of the resting BOLD fluctuation was higher in MWA patients in regions highly related to pain processing and in certain frequencies, the amplitude of the activity of the resting state networks was also higher. While there is no direct evidence that various electrophysiological measures of cortical excitability are represented as a variation in the amplitude or the frequency of the resting BOLD fluctuations, one might speculate that they are strongly related and both depict important features of cortical function.

Several researches investigated the brain resting fMRI activity in migraine with various approaches [17, 19-21, 23, 43, 48, 49]. These approaches are not giving direct information on the cortical hyperexcitability, but a measure of functional interaction. A few studies investigated the amplitude of the resting state BOLD fluctuation in chronic pain conditions $[50,51]$ and found higher amplitude of resting activity in chronic back pain, irritable bowel syndrome, knee osteoarthritis and complex regional pain syndrome. Only single study investigated the amplitude of the low frequency fluctuations in 24 migraineurs, without grouping the patients based on the aura symptoms [52]. Concentrating on only the low frequency component of the BOLD signal (0.01$0.08 \mathrm{~Hz}$ ) they found lower amplitude in migraineus in the cerebellum, bilateral frontal and occipital regions and increased amplitudes in the brainstem and insula. Also our results sustain the well known importance to investigate patients with and without aura symptoms in separate groups. Only the patients experiencing aura symptoms were the ones who had higher amplitude BOLD fluctuation in our study. This was true for the amplitude of the resting activity and also for the amplitude of the activity of the resting state networks.

The importance of the various frequencies of BOLD fluctuations is not yet known but recently a few studies started to explore this feature [53], especially in pain conditions [30, 54]. It was proposed that functional connectivity of various brain regions are represented in different dominant frequency bands [55]. Another option might be that migraine was proposed to be a neurovascular disease, and the altered neurovascular coupling may affect the frequency of the resting BOLD fluctuations [56] by acting as a filter. Furthermore, the group difference in the resting state network activity might well be the result of improved signal to noise ratio by filtering out the low and high frequency artifact. Since most of the slow frequency fluctuation in our analysis with a relatively long TR were shown to be neural origin [57], this hypothesis seems rather unlikely.

Another important aspect of our results is the spatial localisation of the amplitude differences. In the various frequency ranges extensive fronto-parietal and cerebellar areas showed higher activity in MWA patients. Most of these regions were associated with various aspects of the pain processing. Primary and secondary somatosensory cortices, insula, cingulate cortex and prefrontal cortex and subcortical regions, such as the periaqueductal gray matter, hypothalamus, amygdala, hippocampus and cerebellum are commonly activating in response to painful stimuli and referred to as the pain matrix [58]. These key regions were reported to subserve multiple functions 
in pain processing, including sensory discrimination, motivation-affect, motor, attention and also arousal and response selection functions [59].

Cingulate cortex in particular was shown to be one of the key region in experiencing pain [60-62] and it is also part of the descending pain modulatory system [63]. Anterior cingulate cortex is connected to the periaqueductal grey matter, a key region in migraine pathomechanism [64]. In a recent work we also showed that chronic inflammatory pain related sensitization is mediated by the altered activation and connectivity of the cingulate cortex in rats [65].

It was also shown that cerebellum actively contributes to various aspects of pain processing [66]. Subclinical vestibulo-cerebellar dysfunctions were found in migraine with and without aura $[67,68]$. The macro and microstructure of is cerebellum is altered in migraine $[69,70]$. The interictal perfusion of the cerebellum was not found affected by the disease, independently of aura symptoms [71]. In migraine patients Wang and colleagues found altered amplitude of low frequency fluctuation [52], higher and lower amplitudes were found in various parts of the cerebellum. Importantly, in their study no information was given if the patients experienced aura.

The fronto-parietal regions showing increased amplitude of resting activity fluctuation in our study are presumably the same, which are considered to be associated with executive functions. The subclinical executive dysfunction is a known feature of the disease [49, 72, 73]. A recent investigation also described the altered expression of the fronto-parietal resting state network in MWoA patients [49] in highly similar regions what we have found in our analysis.

\section{Conclusion}

In summary, the the regions we found to have increased amplitude of activity fluctuation in MWA patients are those known to be affected by the disease and responsible for the functional alterations also.

\begin{abstract}
Acknowledgement
The authors declare that there are no conflicts of interest. The study was supported by the "Neuroscience Research Group of the Hungarian Academy of Sciences and University of Szeged", the National Brain Research Program (Grant No. KTIA_13_NAP-A-II/20.) and an OTKA [PD 104715] grant. Dr. Szabó was supported by the Bolyai Scholarship Programme of the Hungarian Academy of Sciences.
\end{abstract}

\section{Authors' contributions}

ÁP, JT, DSZ, BT and ZSTK participated in patients recruitment, diagnosis statement and clinical data collection. PF, ZSTK and NSZ participated in the manuscript writing. ET, PF, GCS and AK performed the analysis in the MRI and statistical data. LV and ZSTK supervised and directed the study. All authors read and approved the final manuscript.

\section{Competing interests}

The authors declare that they have no competing interests.

\section{Author details}

${ }^{1}$ Department of Neurology, Neuroimaging Research Group, Albert Szent-Györgyi, Clinical Center, University of Szeged, Semmelweis u. 6, H-6725 Szeged, Hungary. ${ }^{2}$ MTA-SZTE Neuroscience Research Group, Szeged, Hungary. ${ }^{3}$ International Clinical Research Center, St. Anne's University Hospital Brno, Brno, Czech Republic.

Received: 27 October 2016 Accepted: 20 December 2016

Published online: 25 January 2017

\section{References}

1. Lipton RB, Bigal ME, Steiner TJ, Silberstein SD, Olesen J (2004) Classification of primary headaches. Neurology 63:427-435

2. Headache Classification Committee of the International Headache (2013) The international classification of headache disorders, 3rd edition (beta version). Cephalalgia 33:629-808

3. Manzoni GC, Torelli P (2008) Migraine with and without aura: a single entity? Neurol sci 29(Suppl 1):S40-43

4. Ranson R, Igarashi H, MacGregor EA, Wilkinson M (1991) The similarities and differences of migraine with aura and migraine without aura: a preliminary study. Cephalalgia 11:189-192

5. Hadjikhani N, Sanchez Del Rio M, Wu O, Schwartz D, Bakker D, Fischl B, Kwong KK, Cutrer FM, Rosen BR, Tootell RB, Sorensen AG, Moskowitz MA (2001) Mechanisms of migraine aura revealed by functional MRI in human visual cortex. Proc Natl Acad Sci U S A 98:4687-4692

6. Petrusic I, Zidverc-Trajkovic J (2014) Cortical spreading depression: origins and paths as inferred from the sequence of events during migraine aura. Funct Neurol 29:207-212

7. Antal A, Temme J, Nitsche MA, Varga ET, Lang N, Paulus W (2005) Altered motion perception in migraineurs: evidence for interictal cortical hyperexcitability. Cephalalgia 25:788-794

8. Pierelli F, lacovelli E, Bracaglia M, Serrao M, Coppola G (2013) Abnormal sensorimotor plasticity in migraine without aura patients. Pain 154:1738-1742

9. Aurora SK, Ahmad BK, Welch KM, Bhardhwaj P, Ramadan NM (1998) Transcranial magnetic stimulation confirms hyperexcitability of occipital cortex in migraine. Neurology 50:1111-1114

10. Chadaide Z, Arlt S, Antal A, Nitsche MA, Lang N, Paulus W (2007) Transcranial direct current stimulation reveals inhibitory deficiency in migraine. Cephalalgia 27:833-839

11. Gawel M, Connolly JF, Rose FC (1983) Migraine patients exhibit abnormalities in the visual evoked potential. Headache 23:49-52

12. Afra J, Proietti Cecchini A, Sandor PS, Schoenen J (2000) Comparison of visual and auditory evoked cortical potentials in migraine patients between attacks. Clin Neurophysiol 111:1124-1129

13. Brigo F, Storti M, Nardone R, Fiaschi A, Bongiovanni LG, Tezzon F, Manganotti $P$ (2012) Transcranial magnetic stimulation of visual cortex in migraine patients: a systematic review with meta-analysis. J Headache Pain 13:339-349

14. Mantini D, Perrucci MG, Del Gratta C, Romani GL, Corbetta M (2007) Electrophysiological signatures of resting state networks in the human brain. Proc Natl Acad Sci U S A 104:13170-13175

15. Beckmann CF, DeLuca M, Devlin JT, Smith SM (2005) Investigations into resting-state connectivity using independent component analysis. Philos T Roy Soc B 360:1001-1013

16. Xue T, Yuan K, Zhao L, Yu D, Zhao L, Dong T, Cheng P, von Deneen KM, Qin W, Tian J (2012) Intrinsic brain network abnormalities in migraines without aura revealed in resting-state fMRI. PloS One 7(12):e52927. doi:10.1371/journal. pone.0052927

17. Tessitore A, Russo A, Giordano A, Conte F, Corbo D, De Stefano M, Cirillo S, Cirillo M, Esposito F, Tedeschi G (2013) Disrupted default mode network connectivity in migraine without aura. J Headache Pain 14:89. doi:10.1186/ 1129-2377-14-89

18. Yuan $K$, Zhao L, Cheng $P$, Yu DH, Zhao LM, Dong T, Xing LH, Bi YZ, Yang XJ, von Deneen KM, Liang FR, Gong QY, Qin W, Tian J (2013) Altered structure and resting-state functional connectivity of the basal ganglia in migraine patients without aura. J Pain 14:836-844

19. Mainero C, Boshyan J, Hadjikhani N (2011) Altered functional magnetic resonance imaging resting-state connectivity in periaqueductal gray networks in migraine. Ann Neurol 70:838-845

20. Tedeschi G, Russo A, Conte F, Corbo D, Caiazzo G, Giordano A, Conforti R, Esposito F, Tessitore A (2015) Increased interictal visual network connectivity 
in patients with migraine with aura. Cephalalgia: an international journal of headache. doi:10.1177/0333102415584360

21. Hadjikhani N, Ward N, Boshyan J, Napadow V, Maeda Y, Truini A, Caramia F, Tinelli E, Mainero C (2013) The missing link: enhanced functional connectivity between amygdala and visceroceptive cortex in migraine. Cephalalgia 33:1264-1268

22. Niddam DM, Lai KL, Fuh JL, Chuang CY, Chen WT, Wang SJ (2015) Reduced functional connectivity between salience and visual networks in migraine with aura. Cephalalgia: an international journal of headache. doi:10.1177/ 0333102415583144

23. Tessitore A, Russo A, Conte F, Giordano A, De Stefano M, Lavorgna L, Corbo D, Caiazzo G, Esposito F, Tedeschi G (2015) Abnormal connectivity within executive resting-state network in migraine with aura. Headache 55:794-805

24. Hougaard A, Amin FM, Magon S, Sprenger T, Rostrup E, Ashina M (2015) No abnormalities of intrinsic brain connectivity in the interictal phase of migraine with aura. Eur $j$ neurol 22:702-e746

25. Hougaard A, Amin FM, Hoffmann MB, Larsson HB, Magon S, Sprenger T, Ashina M (2015) Structural gray matter abnormalities in migraine relate to headache lateralization, but not aura. Cephalalgia 35:3-9

26. Schwedt TJ, Schlaggar BL, Mar S, Nolan T, Coalson RS, Nardos B, Benzinger T, Larson-Prior $\amalg$ (2013) Atypical resting-state functional connectivity of affective pain regions in chronic migraine. Headache 53:737-751

27. Jin CW, Yuan K, Zhao LM, Zhao L, Yu DH, von Deneen KM, Zhang M, Qin W, Sun WX, Tian J (2013) Structural and functional abnormalities in migraine patients without aura. NMR Biomed 26:58-64

28. Boyacioglu R, Beckmann CF, Barth M (2013) An investigation of RSN frequency spectra using ultra-fast generalized inverse imaging. Front Hum Neurosci 7:156. doi:10.3389/fnhum.2013.00156.eCollection

29. Zou QH, Zhu CZ, Yang Y, Zuo XN, Long XY, Cao QJ, Wang YF, Zang YF (2008) An improved approach to detection of amplitude of low-frequency fluctuation (ALFF) for resting-state fMRI: fractional ALFF. J Neurosci Methods 172:137-141

30. Kim JY, Kim SH, Seo J, Kim SH, Han SW, Nam EJ, Kim SK, Lee HJ, Lee SJ, Kim YT, Chang Y (2013) Increased power spectral density in resting-state pain-related brain networks in fibromyalgia. Pain 154:1792-1797

31. Smith SM (2002) Fast robust automated brain extraction. Hum Brain Mapp 17:143-155

32. Jenkinson M, Bannister P, Brady M, Smith S (2002) Improved optimization for the robust and accurate linear registration and motion correction of brain images. Neuroimage 17:825-841

33. Beckmann CF, Smith SM (2005) Tensorial extensions of independent component analysis for multisubject FMRI analysis. Neuroimage 25:294-311

34. Oelkers R, Grosser K, Lang E, Geisslinger G, Kobal G, Brune K, Lotsch J (1999) Visual evoked potentials in migraine patients: alterations depend on pattern spatial frequency. Brain 122(Pt 6):1147-1155

35. Connolly JF, Gawel M, Rose FC (1982) Migraine patients exhibit abnormalities in the visual evoked potential. J Neurol Neurosurg Psychiatry 45:464-467

36. Diener HC, Scholz E, Dichgans J, Gerber WD, Jack A, Bille A, Niederberger U (1989) Central effects of drugs used in migraine prophylaxis evaluated by visual evoked potentials. Ann Neurol 25:125-130

37. Coppola G, Bracaglia M, Di Lenola D, Di Lorenzo C, Serrao M, Parisi V, Di Renzo A, Martelli F, Fadda A, Schoenen J, Pierelli F (2015) Visual evoked potentials in subgroups of migraine with aura patients. J Headache Pain 16:92

38. Sand T, Zhitniy N, White LR, Stovner LJ (2008) Visual evoked potential latency, amplitude and habituation in migraine: a longitudinal study. Clin Neurophysiol 119:1020-1027

39. Brigo F, Storti M, Tezzon F, Manganotti P, Nardone R (2013) Primary visual cortex excitability in migraine: a systematic review with meta-analysis. Neurol Sci 34:819-830

40. Vincent M, Pedra E, Mourao-Miranda J, Bramati IE, Henrique AR, Moll $J$ (2003) Enhanced interictal responsiveness of the migraineous visual cortex to incongruent bar stimulation: a functional MRI visual activation study. Cephalalgia 23:860-868

41. Noseda R, Burstein R (2011) Advances in understanding the mechanisms of migraine-type photophobia. Curr Opin Neurol 24:197-202

42. Boulloche N, Denuelle M, Payoux P, Fabre N, Trotter Y, Geraud G (2010) Photophobia in migraine: an interictal PET study of cortical hyperexcitability and its modulation by pain. J Neurol Neurosurg Psychiatry 81:978-984

43. Datta R, Aguirre GK, Hu S, Detre JA, Cucchiara B (2013) Interictal cortical hyperresponsiveness in migraine is directly related to the presence of aura. Cephalalgia 33:365-374
44. Cucchiara B, Datta R, Aguirre GK, Idoko KE, Detre J (2015) Measurement of visual sensitivity in migraine: validation of two scales and correlation with visual cortex activation. Cephalalgia 35:585-592

45. Gonzalez de la Aleja J, Ramos A, Mato-Abad V, Martinez-Salio A, HernandezTamames JA, Molina JA, Hernandez-Gallego J, Alvarez-Linera J (2013) Higher glutamate to glutamine ratios in occipital regions in women with migraine during the interictal state. Headache 53:365-375

46. Bridge H, Stagg CJ, Near J, Lau Cl, Zisner A, Cader MZ (2015) Altered neurochemical coupling in the occipital cortex in migraine with visual aura. Cephalalgia 35:1025-1030

47. Sarchielli P, Tarducci R, Presciutti O, Gobbi G, Pelliccioli GP, Stipa G, Alberti A, Capocchi G (2005) Functional 1H-MRS findings in migraine patients with and without aura assessed interictally. Neuroimage 24: 1025-1031

48. Hougaard A, Amin FM, Hoffmann MB, Rostrup E, Larsson HB, Asghar MS, Larsen VA, Olesen J, Ashina M (2014) Interhemispheric differences of fMRI responses to visual stimuli in patients with side-fixed migraine aura. Hum Brain Mapp 35:2714-2723

49. Russo A, Tessitore A, Giordano A, Corbo D, Marcuccio L, De Stefano M, Salemi F, Conforti R, Esposito F, Tedeschi G (2012) Executive resting-state network connectivity in migraine without aura. Cephalalgia 32:1041-1048

50. Ma X, Li S, Tian J, Jiang G, Wen H, Wang T, Fang J, Zhan W, Xu Y (2015) Altered brain spontaneous activity and connectivity network in irritable bowel syndrome patients: a resting-state fMRI study. Clin Neurophysiol 126:1190-1197

51. Baliki MN, Mansour AR, Baria AT, Apkarian AV (2014) Functional reorganization of the default mode network across chronic pain conditions. PLoS One 9:e106133

52. Wang JJ, Chen X, Sah SK, Zeng C, Li YM, Li N, Liu MQ, Du SL (2016) Amplitude of low-frequency fluctuation (ALFF) and fractional ALFF in migraine patients: a resting-state functional MRI study. Clin Radiol 71:558-564

53. Gao L, Bai L, Zhang Y, Dai XJ, Netra R, Min Y, Zhou F, Niu C, Dun W, Gong $H$, Zhang M (2015) Frequency-dependent changes of local resting oscillations in sleep-deprived brain. PLoS One 10:e0120323

54. Otti A, Guendel H, Wohlschlager A, Zimmer C, Noll-Hussong M (2013) Frequency shifts in the anterior default mode network and the salience network in chronic pain disorder. BMC Psychiatry 13:84

55. Salvador R, Martinez A, Pomarol-Clotet E, Gomar J, Vila F, Sarro S, Capdevila A, Bullmore E (2008) A simple view of the brain through a frequency-specific functional connectivity measure. Neuroimage 39:279-289

56. Malinen $\mathrm{S}$, Vartiainen $\mathrm{N}$, Hlushchuk $\mathrm{Y}$, Koskinen M, Ramkumar $\mathrm{P}$, Forss N, Kalso E, Hari R (2010) Aberrant temporal and spatial brain activity during rest in patients with chronic pain. Proc Natl Acad Sci U S A 107:6493-6497

57. Boyacioglu R, Beckmann CF, Barth M (2013) An investigation of RSN frequency spectra using ultra-fast generalized inverse imaging. Front Hum Neurosci 7:156

58. Lee MC, Tracey I (2013) Imaging pain: a potent means for investigating pain mechanisms in patients. Br J Anaesth 111:64-72

59. Davis KD, Moayedi M (2013) Central mechanisms of pain revealed through functional and structural MRI. J Neuroimmune Pharmacol 8:518-534

60. Bliss TV, Collingridge GL, Kaang B-K, Zhuo M (2016) Synaptic plasticity in the anterior cingulate cortex in acute and chronic pain. Nature Reviews Neuroscience

61. Cohen S, Mao J (2014) Neuropathic Pain: Mechanisms \& Clinical Implications + MP3. Br Med J 348:f7656

62. Treede R-D, Kenshalo DR, Gracely RH, Jones AK (1999) The cortical representation of pain. Pain 79:105-111

63. Denk F, McMahon SB, Tracey I (2014) Pain vulnerability: a neurobiological perspective. Nat Neurosci 17:192-200

64. Floyd NS, Price JL, Ferry AT, Keay KA, Bandler R (2000) Orbitomedial prefrontal cortical projections to distinct longitudinal columns of the periaqueductal gray in the rat. J Comp Neurol 422:556-578

65. Spisak T, Pozsgay Z, Aranyi C, David S, Kocsis P, Nyitrai G, Gajari D, Emri M, Czurko A, Kincses ZT, Central sensitization related changes of effective and functional connectivity in the rat inflammatory trigeminal pain model. Neuroscience (in press)

66. Moulton EA, Schmahmann JD, Becerra L, Borsook D (2010) The cerebellum and pain: passive integrator or active participator? Brain Res Rev 65:14-27

67. Sandor PS, Mascia A, Seidel L, de Pasqua V, Schoenen J (2001) Subclinical cerebellar impairment in the common types of migraine: a threedimensional analysis of reaching movements. Ann Neurol 49:668-672 
68. Harno H, Hirvonen T, Kaunisto MA, Aalto H, Levo H, Isotalo E, Kallela M, Kaprio J, Palotie A, Wessman M, Farkkila M (2003) Subclinical vestibulocerebellar dysfunction in migraine with and without aura. Neurology 61:1748-1752

69. Granziera C, Romascano D, Daducci A, Roche A, Vincent M, Krueger G, Hadjikhani N (2013) Migraineurs without aura show microstructural abnormalities in the cerebellum and frontal lobe. Cerebellum 12:812-818

70. Jin C, Yuan K, Zhao L, Zhao L, Yu D, von Deneen KM, Zhang M, Qin W, Sun W, Tian J (2013) Structural and functional abnormalities in migraine patients without aura. NMR Biomed 26:58-64

71. Arkink EB, Bleeker EJ, Schmitz N, Schoonman GG, Wu O, Ferrari MD, van Buchem MA, van Osch MJ, Kruit MC (2012) Cerebral perfusion changes in migraineurs: a voxelwise comparison of interictal dynamic susceptibility contrast MRI measurements. Cephalalgia 32:279-288

72. O'Bryant SE, Marcus DA, Rains JC, Penzien DB (2005) Neuropsychology of migraine: present status and future directions. Expert Rev Neurother 5:363-370

73. Camarda C, Monastero R, Pipia C, Recca D, Camarda R (2007) Interictal executive dysfunction in migraineurs without aura: relationship with duration and intensity of attacks. Cephalalgia 27:1094-1100

\section{Submit your manuscript to a SpringerOpen ${ }^{\circ}$ journal and benefit from:}

- Convenient online submission

- Rigorous peer review

- Immediate publication on acceptance

Open access: articles freely available online

- High visibility within the field

- Retaining the copyright to your article

Submit your next manuscript at $\boldsymbol{~ s p r i n g e r o p e n . c o m ~}$ 